\title{
Use of a lipid nanoparticle system as a Trojan horse in delivery of gold nanoparticles to human breast cancer cells for improved outcomes in radiation therapy
}

\author{
Kyle Bromma ${ }^{1}$, Kristy Rieck' , Jayesh Kulkarni ${ }^{2,3}$, Connor O'Sullivan ${ }^{4}$, Wonmo Sung ${ }^{5}$, Pieter Cullis ${ }^{2}$, \\ Jan Schuemann ${ }^{5}$ and Devika B. Chithrani ${ }^{1,6^{*}}$ (]
}

${ }^{*}$ Correspondence:

devikac@uvic.ca

${ }^{1}$ Department of Physics

and Astronomy, University

of Victoria, Victoria, BC,

Canada

Full list of author information is available at the end of the article

\begin{abstract}
Background: Radiotherapy is commonly used for treating cancer. Novel sensitizers, such as gold nanoparticles (GNPs), are being used to enhance the local radiation dose. It is not known how the uptake and radiation dose enhancement of GNPs vary in synchronized vs unsynchronized (control) tumor cell populations. Successful application of GNPs in radiation therapy requires NPs to be accumulated within individual tumor cells at clinically feasible NP concentrations. Use of small GNPs as a radiation dose enhancer in the past required very high NP concentration, since the driving force for the uptake of smaller GNPs is low. We used a novel lipid-based NP of $50 \mathrm{~nm}$ diameter system as a Trojan horse to deliver smaller GNPs of size $5 \mathrm{~nm}$ (LNP-GNP) at $0.2 \mathrm{nM}$ concentration. We investigated the changes in GNP uptake and survival fraction with the LNP delivery at different cell stages using human breast cancer as our tumor model and choosing the triple-negative MDA-MB-231 cell line.
\end{abstract}

Results: Using the LNP-GNP system resulted in a 39- and 73-fold enhancement in uptake of $5 \mathrm{~nm}$ GNPs in unsynchronized and synchronized tumor cell populations, respectively. The NP uptake per cell increased from 800 to 1200 and from 30,841 to 88,477 for individual $5 \mathrm{~nm}$ GNPs and $5 \mathrm{~nm}$ GNPs incorporated in LNPs, respectively. After a radiation dose of $2 \mathrm{~Gy}$ with $6 \mathrm{MeV}$ photons, synchronized tumor cell populations incorporated with LNP-GNPs produced a $27 \%$ enhancement in tumor cell death compared to the control (unsynchronized; no GNPs; 2 Gy). The findings of our experimental results were supported by modeling predictions based on Monte Carlo calculations.

Conclusions: This study clearly shows that the cell cycle, GNPs, and radiation therapy can be combined to improve outcome of cancer therapy. Using the experimental data, we estimated the predicted improvement for a clinical treatment plan where 30 fractions of 2 Gy radiation dose were given over a period of time. Enhanced uptake and radiation sensitivity of a synchronous tumor cell population would produce a significant improvement in cell killing. For example, synchronizing cells and the addition of LNP-GNPs into tumor cells produced a 1000-fold enhancement in cell killing. Because the agents used for cell synchronization are in clinical practice, this approach may be a 
simple and cost-effective way to further enhance local radiation dose. Finally, this study provides a novel lipid-based NP platform to further improve GNP-mediated radiation therapy through synchronization of breast cancer cell population.

Keywords: Gold nanoparticles, Radiation therapy, Synchronization, Monte Carlo simulation, DNA damage, Optical imaging, Lipid nanoparticles

\section{Introduction}

According to the World Health Organization, cancer is the second leading cause of death globally and was responsible for an estimated 9.6 million deaths in 2018 . Globally, about one in six deaths is due to cancer. More than half of the cancer patients will receive radiation therapy as part of their treatment plan (Chang et al. 2014). Radiotherapy is a good alternative to surgery for long-term control for many tumors in the head and neck, lung, cervix, bladder, prostate and skin. In the case of breast cancer, post-operative radiotherapy is frequently delivered to the breast and regional lymph nodes (Arthur et al. 2016). However, radiation therapy presents the challenge of delivering a dose to tumor cells, while sparing surrounding normal tissue, through which ionizing radiation must inevitably pass. To help overcome this limitation, the introduction of high atomic number $(\mathrm{Z})$ materials, such as gold nanoparticles (GNPs) as radiation dose enhancers into current radiation therapy protocol is being studied to improve the local therapeutic effects (Cho and Krishnan 2013; Chithrani et al. 2010; Hainfeld et al. 2004; Butterworth et al. 2013). High-Z metal NPs such as gold absorb more X-rays, releasing more electrons, which in turn interact with water molecules present in cells and tissues to produce radicals (O'Neill 1987; Carter et al. 2007). Although most studies suggest that deoxyribonucleic acid (DNA) is damaged indirectly by hydroxyl radicals, electrons can also cause damage to DNA directly (Boudaiffa et al. 2000). Recent in vitro and in vivo studies have shown that the incorporation of GNPs into current radiotherapy protocols produces better outcomes at clinically relevant megavoltage energies (Cruje et al. 2015; Wolfe et al. 2015). Intravenously injected NPs can accumulate within the tumor using its leaky vasculature, penetrate through the tumor tissue and finally enter the individual tumor cells (Abolfazli et al. 2015; Yang et al. 2018). However, since the GNP accumulation and radiation enhancement effect can be different based on the particular tumor microenvironment, NP-based therapeutics need to be tested in many different tumor models.

Lipid-based NP platforms have been proposed for use in improved cancer chemotherapy (Kang and Ko 2015). Incorporation of GNPs into such a lipid-based NP system would facilitate future chemoradiation therapy initiatives (Yang et al. 2018). Lipid nanoparticles (LNPs) are made up of natural lipids, usually phospholipids and cholesterol, which can encapsulate molecules in a hydrophilic or hydrophobic core. In this study, we used an LNP system as a Trojan horse to deliver smaller GNPs (LNP-GNP). A pioneering study by Hainfeld et al. demonstrated that a very high concentration of small GNPs $(1.9 \mathrm{~nm}$ in diameter) was necessary to produce a significant therapeutic effect (Hainfeld et al. 2004). Small GNPs (1-10 nm) are not internalized well by cells on their own, as the mechanism of internalization seems to favor larger particles with the highest uptake 


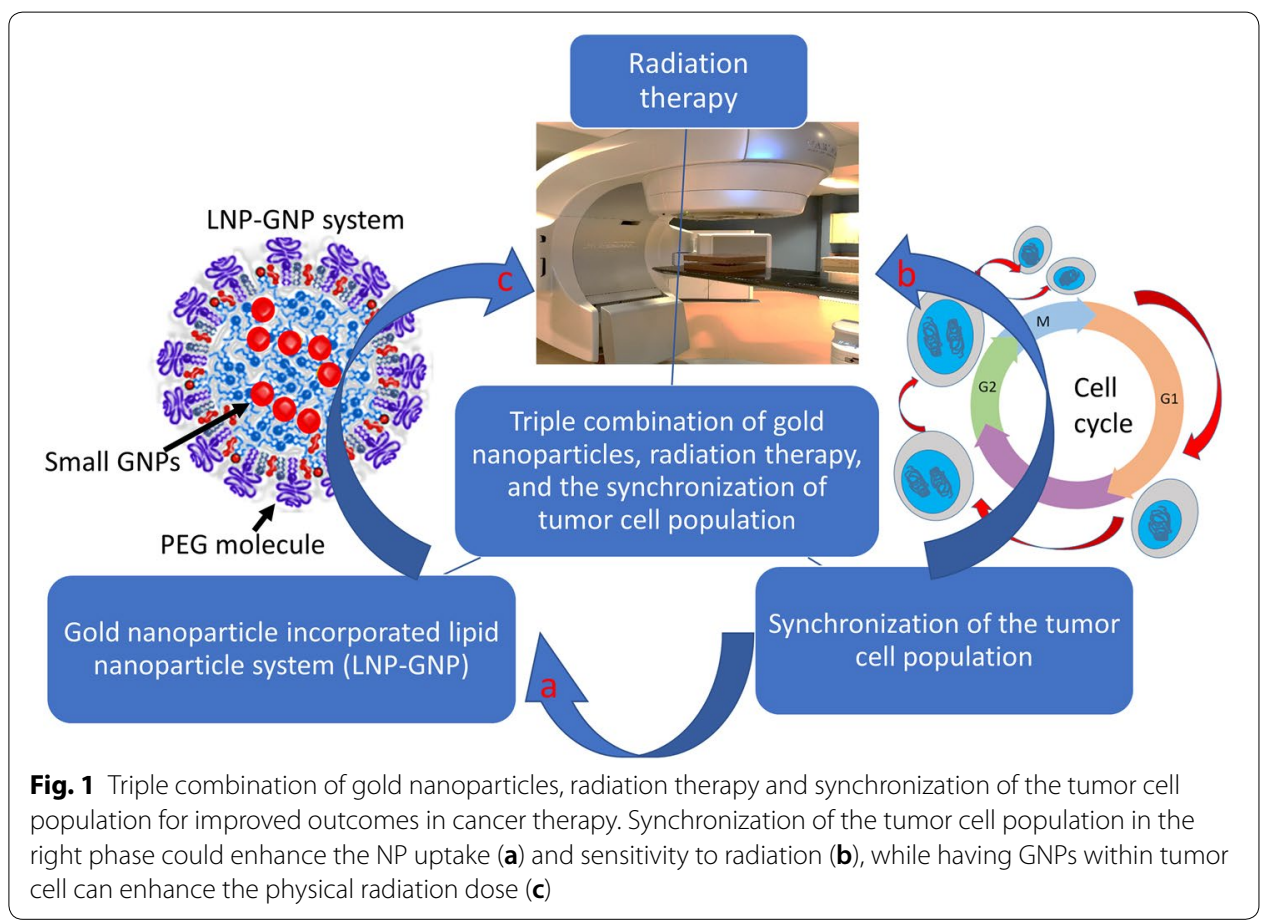

occurring for particles with a diameter of $50 \mathrm{~nm}$ (Chithrani et al. 2006). Lipid encapsulation of metallic nanoparticles is a good way to increase their internalization and biocompatibility, as the surface chemistry of lipids is similar to structural components of the cellular membrane (Von White et al. 2012). LNPs are also of interest as a carrier for gene and drug delivery (Baowan and Thamwattana 2014). Finally, LNPs provide new opportunities to encapsulate both GNPs and anticancer drugs in one vehicle for future combined therapeutics (Lee et al. 2012).

Although GNPs are being explored as a radiation dose enhancer in cancer therapy, it is not yet known how radiation dose enhancement properties vary in a synchronized vs. an unsynchronized tumor cell population. There are tools available in clinics to synchronize tumor cell populations if necessary. We used thymidine to synchronize tumor cell populations in $\mathrm{S}$ phase in vitro, since it is a clinically available pharmacological agent (Heinemann et al. 2010). As illustrated in Fig. 1, this study looked at how the combination of GNPs and synchronization of the tumor cell population affects the final outcome in radiation therapy for the first time using a novel lipid-based NP platform in human breast cancer cells. Our final goal was to compare our experimental data with theoretical predictions based on Monte Carlo simulations using TOPAS-nBio (Schuemann et al. 2019).

Breast cancer $(\mathrm{BC})$ has a high incidence and is the most frequently diagnosed cancer, ranking as the second leading cause of cancer death in women. Triple-negative BC (TNBC) accounts for about $12 \%-17 \%$ of breast cancer cases. TNBC is more aggressive and is associated with a worse prognosis, a higher risk of relapse and metastasis as well as a shorter survival time (DeSantis et al. 2017). Hence, we chose a TNBC cell line, MDA-MB-231 as our in vitro cell model for this study. In addition, this study will provide guidelines to further improve current cancer therapeutics through the inclusion of 
both cell cycle synchronization and GNP delivery into current radiation therapy protocols for the first time as outlined in Fig. 1. Finally, this study unveils a new NP platform for triple combination of chemotherapy, radiation therapy and GNPs in future cancer therapy.

\section{Materials and methods}

\section{Lipid nanoparticle synthesis and analysis}

GNPs of $5 \mathrm{~nm}$ diameter were purchased from Ted Pella, Inc. (Redding, CA) and were entrapped in lipid nanoparticles (LNPs) using a rapid-mixing method (Leung et al. 2015). Lipids [composition: DLin-MC3-DMA/DSPC/cholesterol/PEG-lipid $(50 / 10 / 39 / 1 \mathrm{~mol} \%)]$ were first dissolved in ethanol at $20 \mathrm{mM}$ concentration. GNPs were suspended in $25 \mathrm{mM}$ sodium acetate buffer (pH4) to a ratio of $2.2 \times 10^{13} \mathrm{GNP} / \mu \mathrm{M}$ lipid for LNP formulation. The organic phase and aqueous phase were mixed through a T-junction mixer (PEEK 0712) at a flow rate ratio of $1: 3 \mathrm{v} / \mathrm{v}$ (or $5 \mathrm{~mL} / \mathrm{min}$ ethanol and $15 \mathrm{~mL} / \mathrm{min}$ aqueous). The resulting suspension was then dialyzed into phosphatebuffered saline (PBS) and concentrated $\sim$ fourfold following dialysis. This process forms LNPs that no longer have a typical bilayer structure, but rather an electron-dense core, a significant fraction of the lipids are contained within the particle's core (Kulkarni et al. 2018). To analyze the formulation, LNPs were passed through a VivaPure D Mini H spin column (Sartorius, Goettingen, Germany) pre-equilibrated with PBS. GNP entrapment was measured as a function of the gold-to-lipid ratio before and after the anionexchange column. Lipid content was measured using the Cholesterol E assay kit (Wako Chemicals, Richmond, VA) to determine the amount of cholesterol in the formulation and extrapolate the total lipid content. Particle sizing was performed by dynamic light scattering with a Malvern Zetasizer NanoZS (Worcestershire, UK).

\section{Cellular uptake study}

MDA-MB-231 cells were purchased from ATCC (2014) and maintained in Dulbecco's modified Eagle's medium (HyClone) supplemented with $10 \%$ fetal bovine serum (Gibco). Cells were cultivated in $175 \mathrm{~cm}^{2}$ flasks in a humidified incubator; once $80-90 \%$ confluency was reached, cells were passed to maintain exponential growth. Cells were authenticated for MDA-MB-231 human cell line and tested to confirm the absence of mycoplasma at Charles River research laboratories (MA, USA; May 2016) before initiation of the experiments discussed in this manuscript. Cells were also tested using a fluorescence-based technique every 4 months for mycoplasma contamination during the period of experiments (2017-2018) discussed in this paper (Fischer Scientific; Invitrogen $^{\mathrm{TM}}$ LSM7006).

For quantification and imaging studies, cells were plated in six-well dishes without and with glass coverslips for quantification and optical imaging studies, respectively. Once the confluency within the wells reached 70\%, cells were incubated with NPs at a concentration of $0.2 \mathrm{nM}$ for $20 \mathrm{~h}$. For determining the NPs within cells, the cells were washed three times with PBS after incubation with NPs, followed by adding trypsin to remove cells from the dish for quantification study as described in the next section. For imaging study, the wells with coverslips were rinsed twice with PBS after NP incubation, followed by fixation with $4 \%$ paraformaldehyde (PFA) in PBS for $10 \mathrm{~min}$ at room temperature. The 
coverslips were washed gain two times with PBS to remove fixatives, followed by drying before mounting onto glass slides for microscopy.

\section{GNP uptake quantification}

The cells were collected into a single cell suspension using trypsin $0.25 \%$ (HyClone) as mentioned in the previous section. The concentration of cells was determined using Beckman Coulter Z2 Particle Counter and Size Analyzer. For the inductively coupled plasma mass spectroscopy (ICP-MS) analysis, the cells were digested using Aqua Regia in 3:1 ratio $\mathrm{HCl}: \mathrm{HNO}_{3}$, in an oil bath at $200{ }^{\circ} \mathrm{C}$ for an hour. The samples were measured with the Thermo X-Series II (X7) quadrupole ICP-MS instrument to quantify the amount of gold present. The number of GNPs per cell was calculated as described in our previous publications (Cruje et al. 2015).

\section{Imaging of GNPs using CytoViva microscopy}

This imaging system was designed so that despite NP interaction with cells or tissue, their spectra may still be confirmed because they are still optically observable. The microscope is a dark-field imaging system that uses oblique angle lighting. The result provides high signal-to-noise ratio optimized dark-field-based images. NPs appear bright due to high scattering cross sections of GNPs. To confirm the spectra of GNPs, SAM (Spectral Angle Mapping) was conducted with the CytoViva hyperspectral imaging system. SAM determines the presence of GNPs in the input image by comparing unknown spectra in the acquired hyperspectral image to a user-defined spectrum, which is that of a GNP in these experiments.

\section{Radiation treatment}

After incubation with NPs for $20 \mathrm{~h}$, a 2 Gy radiation with $6 \mathrm{MV}$ photons was given using a linear accelerator (Elekta Oncology Systems, Stockholm, Sweden). A field size of $40 \mathrm{~cm} \times 40 \mathrm{~cm}$ was used so that all the wells were irradiated with 2 Gy at a dose rate of $600 \mathrm{MU} / \mathrm{min}$ at the same time. The survival fractions of irradiated samples were compared to non-irradiated samples using clonogenic assay and DNA double-stand breaks (DSBs) assay as discussed later. A radiation dose of 2 Gy was chosen for two reasons: (a) it is one of the doses used in standard fractionated treatments in the clinic and (b) a higher radiation dose can saturate the induced DSBs making it difficult to determine changes in DNA damage due to the differences in treatment.

\section{Survival fractions of irradiated cells (clonogenic assay)}

After the radiation treatment, the cells were trypsinized and diluted to form single cell suspensions. The required volumes of cell suspension solution were calculated for the control and treatment samples. The calculated volume of cell suspension for each condition was seeded in $60 \mathrm{~mm}$ tissue culture dishes in triplicate. 200 cells were plated for unirradiated conditions and 400 cells for those that received 2 Gy. The cells were left in a $37{ }^{\circ} \mathrm{C}$ humidified incubator with $5 \% \mathrm{CO}_{2}$ for 14 days for colonies to grow. Then, the dishes were stained and fixed with $0.1 \%$ of methylene blue (BioShop) in $70 \%$ ethyl alcohol for $1 \mathrm{~h}$. The stained dishes were rinsed in lukewarm water and left to air dry 
overnight. The air-dried control dishes were then counted. Colonies were defined as structures containing $>50$ cells.

The plating efficiency $(\mathrm{PE})$ was obtained though the following formula:

$$
\mathrm{PE}=\frac{\text { number of colonies counted }}{\text { number of cells plated }}
$$

The colonies of treatment samples were also counted and the survival fraction (SF) was obtained with the following equation:

$$
\mathrm{SF}=\frac{\text { number of colonies counted }}{\text { number of cells plated } \times \mathrm{PE}} \text {. }
$$

\section{Immunofluorescence assay}

The cells were grown on glass coverslips in six-well dishes and incubated with NPs at $0.2 \mathrm{nM}$ concentration for about $20 \mathrm{~h}$ before treating with a radiation dose of 2 Gy with $6 \mathrm{MV}$ photons using a linear accelerator setup. After $24 \mathrm{~h}$ of the treatment, cells were fixed with $2 \%$ paraformaldehyde/ $0.2 \%$ Triton-X 100 for $20 \mathrm{~min}$ at room temperature (Sigma-Aldrich) and washed with PBS three times after the fixation. The cells were then treated with $0.1 \%$ Triton-X for $20 \mathrm{~min}$ at room temperature and washed three times in PBS for 5 min. Cells were treated with 3\% BSA (bovine serum albumin) for $1 \mathrm{~h}$ and coverslips were then placed face down on Para film with a $50 \mu \mathrm{L}$ drop of primary antibody 1:800 (gammaH2AX Ser 139. Millipore 05-363 Lot 2276332) in $3 \% \mathrm{BSA} / \mathrm{PBS}$ and incubated overnight at $4{ }^{\circ} \mathrm{C}$. Coverslips were washed with $0.5 \%$ BSA/0.175\% Tween 20/PBS 3 times for 5 min. Secondary antibody was diluted 1:500 (anti-mouse IgG Alexa 647. Life Technologies Ref A31571, Lot423849) in 3\% BSA/ PBS and coverslips incubated with secondary antibody for 45 min followed by washing with $0.5 \%$ BSA $/ 0.175 \%$ Tween 20/PBS 3 times for 5 min, then with PBS three times for $5 \mathrm{~min}$. Finally, coverslips were dried and mounted on microscope glass slides for imaging.

\section{Synchronization of cells}

The normal cell cycle can be divided into G1, S, G2, and M phases, and cell populations can be synchronized in different phases depending on the method. In this study, we tested two methods for synchronization of a tumor cell population: double thymidine blocking and serum starvation.

\section{Double thymidine blocking}

Thymidine inhibits DNA synthesis and arrests cells in S phase. However, a double thymidine block ensures that any cells that were in mid or late $\mathrm{S}$ phase during the first block will be captured in late G1 or early S phase in the second block. MDA-MB-231 cells were seeded in a six-well plate at $40 \%$ confluency $\left(1.3 \times 10^{5}\right.$ cells). Once the cells were settled and adherent to the dish, they were washed with PBS, followed by adding $3 \mathrm{~mL}$ of $2 \mathrm{mM}$ thymidine in Dulbecco's modified Eagle medium (DMEM) $+10 \%$ fetal bovine serum $(\mathrm{FBS})+1 \%$ penicillin/streptomycin (PS). After $26 \mathrm{~h}$, each well was washed twice 
with PBS and regular DMEM for $16 \mathrm{~h}$. Following the wash, the second thymidine block was carried out using $2 \mathrm{mM}$ thymidine DMEM $+10 \% \mathrm{FBS}+1 \% \mathrm{PS}$ for $24 \mathrm{~h}$ for synchronization of the cell population in S phase.

\section{Serum starvation method for synchronization of cells}

Serum starvation arrests most of the cells in the G0 phase of the cell cycle after an appropriate deprivation period. MDA-MB-231 cells were cultivated in six-well dishes at $40 \%$ confluency $\left(1.3 \times 10^{5}\right.$ cells $)$ in a mixture of DMEM and $10 \%$ FBS. Once the cells had adhered, the media with 10\% FBS was aspirated, cells were washed with PBS and media with $0.5 \%$ FBS (starvation media) was added. The cells were left to incubate in the starvation media for $24 \mathrm{~h}$ or $48 \mathrm{~h}$. After the incubation period, the cells were released into cell cycle by replacing $0.5 \%$ FBS media with $10 \%$ FBS media, and any subsequent experiments were performed.

\section{Preparation of cells for cell cycle analysis}

Synchronization of the population was verified using flow cytometry and propidium iodide staining. At desired time points, cells were harvested and a single cell suspension was prepared in buffer (PBS $+2 \% \mathrm{FBS}$ ). Cells were washed with PBS and centrifuged at $300 \times g$ for 5 min twice. The cell pellet was then fixed by re-suspension in 1\% PFA/ PBS and incubation on ice for $15 \mathrm{~min}$. Cells were again washed in PBS and centrifuged at $350 \times g$ for $5 \mathrm{~min}$. Cells were re-suspended in $0.6 \mathrm{~mL} \mathrm{PBS}$ and $1.4 \mathrm{~mL}$ freezer cold $100 \%$ ethanol (overall $70 \%$ ethanol). Samples were incubated in the dark at $4{ }^{\circ} \mathrm{C}$ for at least an hour to further fix and dehydrate the cell sample. Samples were then centrifuged at $350 \times g$ for $10 \mathrm{~min}$ at $20^{\circ} \mathrm{C}$. The cell pellet was re-suspended in $1 \mathrm{~mL}$ PBS/BSA (0.5\% BSA-bovine serum albumin) and centrifuged at $350 \times g$ for $5 \mathrm{~min}$ at $20{ }^{\circ} \mathrm{C}$. To permeabilize the cell membrane and degrade RNA, the cell pellet was re-suspended in PBTB (PBS, $0.5 \%$ BSA, $0.1 \%$ Triton-X 100) followed by addition of RNaseA (100 $\mu \mathrm{g} / \mathrm{mL})$. Samples were left to shake at $37^{\circ} \mathrm{C}$ for $25 \mathrm{~min}$. For labeling DNA, tubes were covered in foil, propidium iodide (PI) added (1:100 from $1 \mathrm{mg} / \mathrm{mL})$ and incubated on a shaker at $4{ }^{\circ} \mathrm{C}$ for at least $1 \mathrm{~h}$. The cells were then centrifuged at $350 \mathrm{~g}$ for $5 \mathrm{~min}$ at $20^{\circ} \mathrm{C}$. Finally, we re-suspended PI stained cells in $1 \mathrm{~mL}$ of PBS/BSA and passed the solution through a $50 \mu \mathrm{m}$ cell strainer before running on a BD FACScalibur flow cytometer. Propidium iodide binds to DNA and is highly fluorescent at $488 \mathrm{~nm}$ with broad emission centered around $600 \mathrm{~nm}$. Synchronization was verified by analyzing the amount of DNA content in cell populations, as this indicates which phase the cell population is in.

\section{Theoretical estimation of dose enhancement using Monte Carlo simulations}

The local dose enhancement and the expected decrease in cell survival were estimated using Monte Carlo simulations (Lin et al. 2015; Sung et al. 2017). In short, radial dose distributions of secondary electrons were calculated for single GNPs irradiated by a $6 \mathrm{MV}$ photon spectrum from a Varian TrueBeam using a phase space provided on the Varian website as particle source for simulations in the TOPAS-nBio Monte Carlo system version 
3.0.1 (Schuemann et al. 2019). The radial dose distributions were then used as input to a modified local effect model approach for GNPs (GNP-LEM), which was used to calculate the number of lethal damages in the cell nucleus for a given GNP distribution inside and around a cell (Lin et al. 2015). MDA-MB-231 cells were represented by a $2 \mu$ m-thick ellipsoid with minor/major axis diameters of 8.5/18.5 $\mu \mathrm{m}$ (Liu et al. 2015). To calculate survival fractions, we used the parameters for the linear quadratic model $\alpha=0.019$ and $\beta=0.052$ (Jain et al. 2011). Our current GNP-LEM approach does not include changes in the cell shape and radiosensitivity for different cell phases. Instead, we consider the difference between synchronized vs. unsynchronized cell populations by using the different number of GNPs in the cells. We accounted for both uncertainties of GNP uptake measurement and statistical uncertainty in the model.

\section{Results}

\section{Characterization of LNP-GNP complexes}

Transmission electron microscopy (TEM) image of larger LNPs incorporated with smaller GNPs is displayed in Fig. 2a. Based on TEM images, the diameter of smaller GNPs and LNP-GNPs were $4.6 \pm 0.5$ and $53.3 \pm 8.6$, respectively. For convenience, we will refer to the LNP-GNPs as "50 nm LNP-GNPs" and to smaller GNPs as " $5 \mathrm{~nm}$ GNPs". A TEM image of $5 \mathrm{~nm}$ GNPs used for encapsulation in LNPs is given in Additional file 1: Fig. S1A. The surface plasmon absorption was measured using

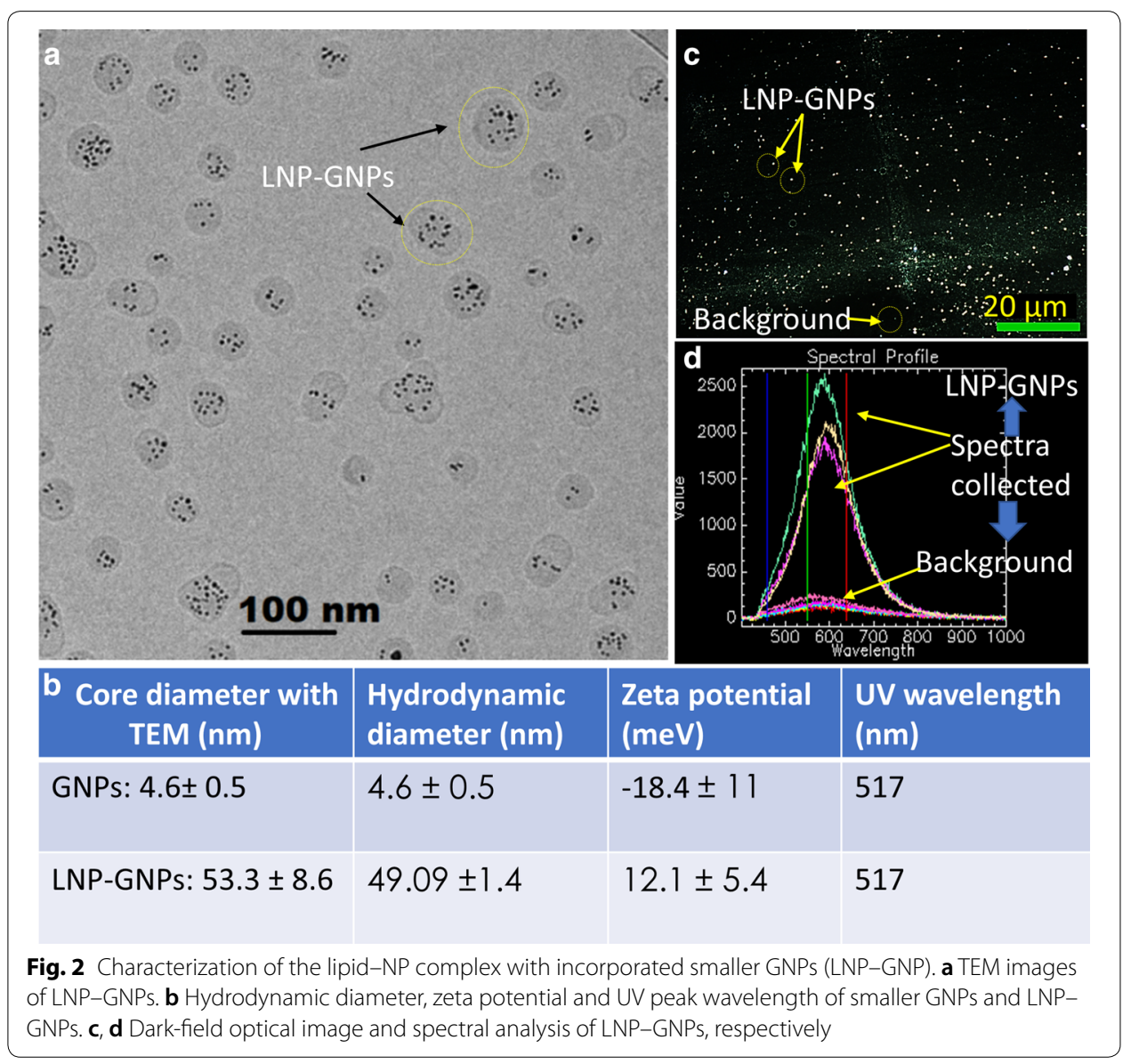


ultraviolet-visible (UV-Vis) spectroscopy to further verify the presence of GNPs, their size, and homogeneity (see Fig. 2b, Additional file 1: Fig. S1B). The LNP-GNP system had a peak wavelength of $517 \mathrm{~nm}$ which coincides with the peak of $5 \mathrm{~nm}$ GNPs. This confirms that $5 \mathrm{~nm}$ GNPs were incorporated into the lipid-NP system and that smaller GNPs did not aggregate during the encapsulation process. The TEM image of LNP-GNPs (Fig. 2a) further confirms the stability of $5 \mathrm{~nm}$ GNPs within LNPs. NPs were further characterized by measuring their hydrodynamic diameter and zeta potential as listed in Fig. 2b. Cytoviva's enhanced dark-field and hyperspectral imaging (HSI) were used to optically observe and achieve spectral mapping of NPs. Figure 2c, d shows the dark-field image and spectra collected from selected LNP-GNPs in their corresponding HSI image, respectively.

\section{Cellular uptake of NP complexes in MDA-MB-231 breast cancer cells}

The endolysosomal pathway of NPs within a tumor cell is shown in Fig. 3a. The uptake of NPs is dependent on their size. Individual $5 \mathrm{~nm}$ GNPs had a lower uptake as compared to the ones incorporated in the LNP system (see Fig. 3b). In this study, we were able to use $50 \mathrm{~nm}$-diameter LNPs as a driving force to deliver small GNPs $(5 \mathrm{~nm})$ and a 39-fold increase in uptake was observed. In addition, we were able to increase the biocompatibility of $5 \mathrm{~nm}$ GNPs by incorporating them into LNPs (Additional file 1: Fig. S2). We used Cytoviva's enhanced dark-field and hyperspectral imaging (HSI) to optically observe and spectrally map NPs within cells. Dark-field images and reflectance spectra collected from a few NPs clusters in the corresponding HSI image are shown in Fig. 3c, d, respectively. Optical images of cells with and without $5 \mathrm{~nm}$ GNPs are shown in Additional file 1: Fig. S3.
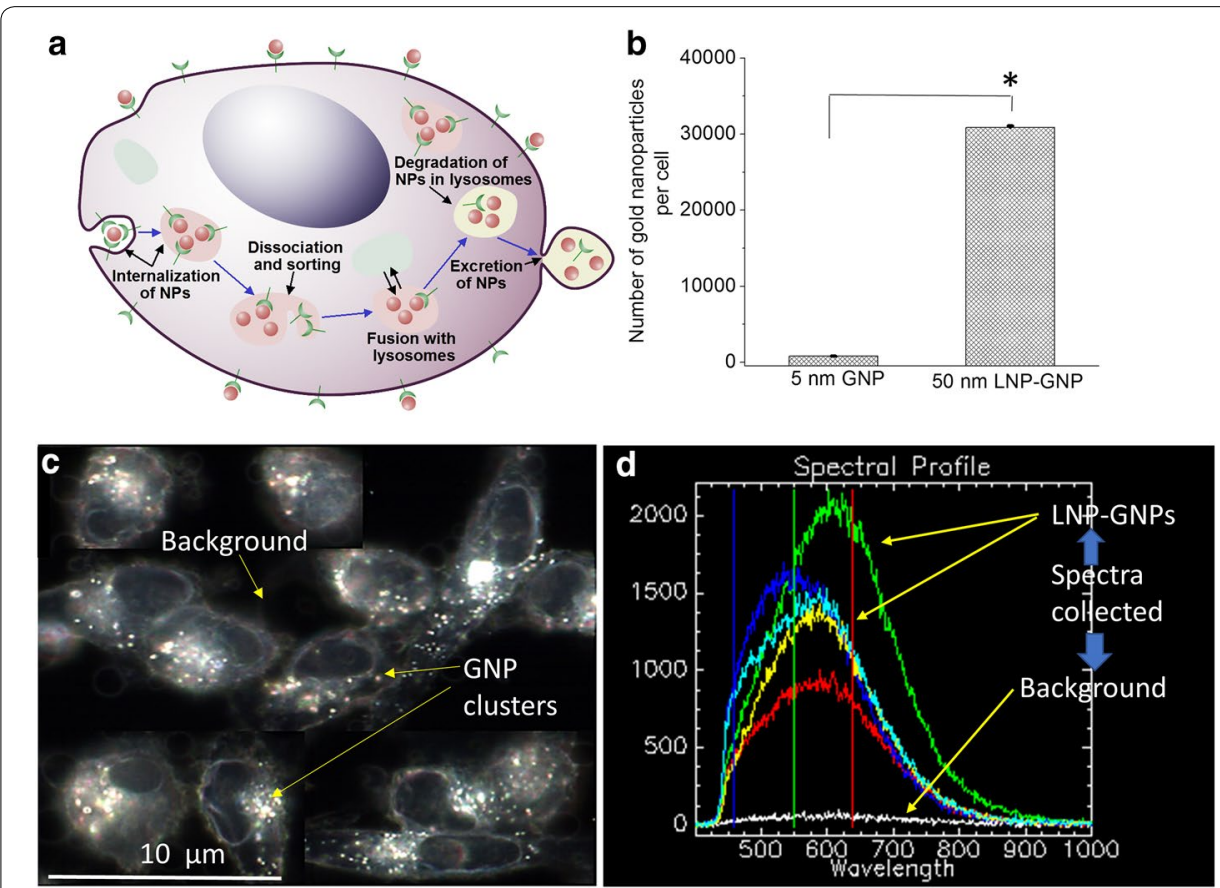

Fig. 3 Cellular uptake of NP complexes. a Endolysosomal pathway of NPs within cells. b Cellular uptake data for $5 \mathrm{~nm}$ GNPs and $50 \mathrm{~nm}$ LNP-GNPs. Data are "mean \pm SD" for $n=3$. *Represents a statistically significant difference (unpaired $t$ test, $p<0.05$ ). $\mathbf{c}, \mathbf{d}$ Dark-field image and a few spectra collected from NP clusters in the corresponding HSI image, respectively. The bright dot-like structures are GNP clusters localized within cells 


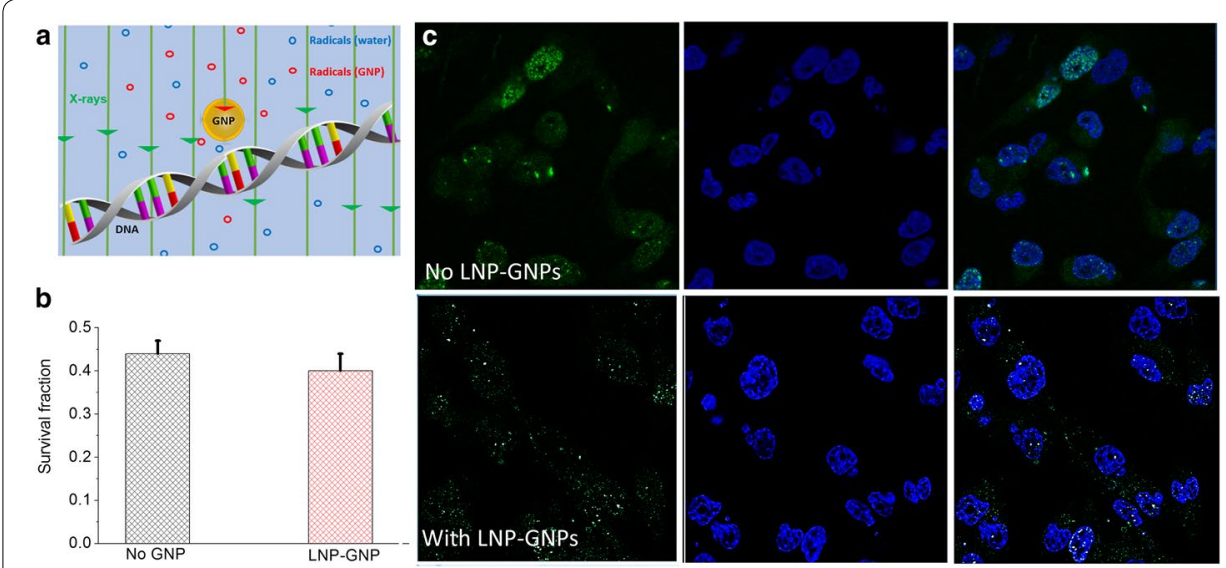

Fig. 4 GNP-mediated radiation therapy. a Schematic showing formation of harmful species, such as free radicals, when incoming radiation interacts with GNPs. $\mathbf{b}$ Experimental survival fraction of cells treated with a 2 Gy radiation dose. Data are "mean $\pm S D$ " for $n=3$. c Probing of DNA DSBs $24 \mathrm{~h}$ after radiation treatment to assess the effect of radiation treatment (left most panel: nuclei marked in blue; middle panel: $\gamma \mathrm{H} 2 \mathrm{AX}$ foci marked in green; and right most panel: merged image)

\section{Radiation dose enhancement due to LNP-GNPs}

One of the mechanisms of increase in cell death is illustrated in Fig. 4a, where the presence of GNPs could produce more free radicals leading to more damage in DNA. The survival fraction was lower for cells treated with LNP-GNPs (see Fig. 4b). We also probed the DNA DSBs to measure the radiation-induced damage as seen in Fig. 4c. However, there was no significant difference in DNA damage in cells treated with LNP-GNPs after treatment with radiation. This is consistent with the results from the clonogenic assay. The linear accelerator setup used for the radiation experiment is shown in Additional file 1: Fig. S4. To further increase the NP uptake and improve the therapeutic outcome, we synchronized the tumor cell population as discussed in the next few sections.

\section{Cellular uptake of GNPs in synchronized vs. control (unsynchronized) cell population}

To synchronize cell populations, we tested both the double thymidine block method and the serum starvation method. We discovered that the double thymidine block method was more effective in synchronizing a cell population as compared to the serum starvation method (see Additional file 1: Fig. S5). We checked the progression of synchronized cell population as a function of time (see Fig. 5a). Three hours after cell synchronization, cells were incubated with NPs for $20 \mathrm{~h}$ at a concentration of $0.2 \mathrm{nM}$. As illustrated in Fig. 5b, using the LNP-GNP system resulted in a 73-fold enhancement in uptake of $5 \mathrm{~nm}$ GNPs for synchronized tumor cell populations. The NP uptake per cell increased from 800 to 1200 and from 30,841 to 88,477 for individual $5 \mathrm{~nm}$ GNPs and $5 \mathrm{~nm}$ GNPs incorporated in LNPs, respectively. Based on this information, we used a double thymidine block method to evaluate the change in the radiation dose enhancement in a synchronized vs. unsynchronized cell population. 

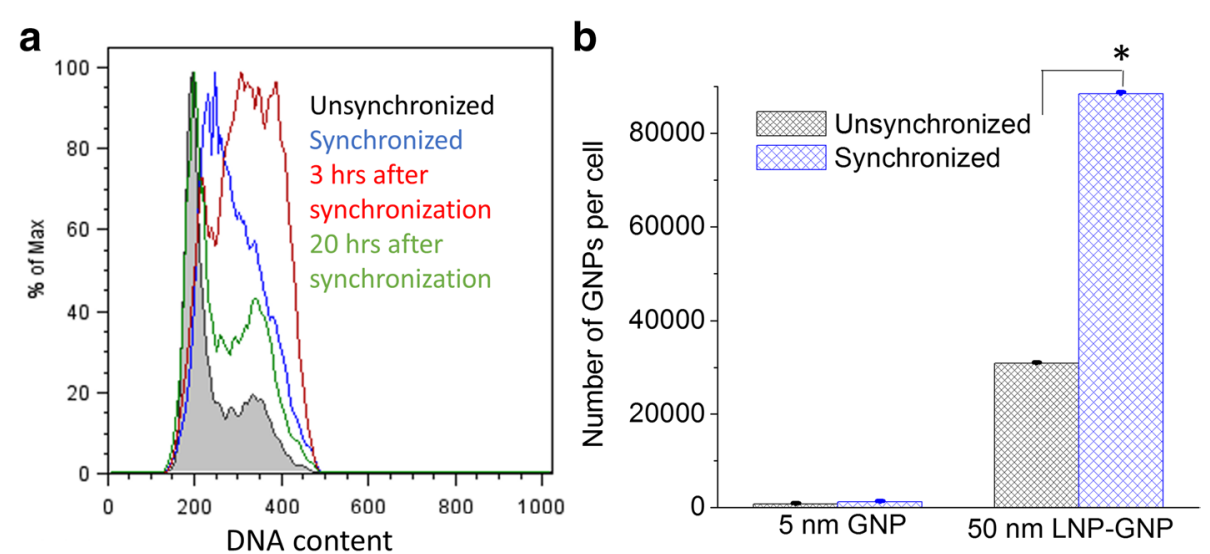

Fig. 5 Cellular uptake of NP complexes in synchronized vs. unsynchronized tumor cell populations. a Progression through the cell cycle after synchronization using the double thymidine blocking method. $\mathbf{b}$ Cellular uptake data of $5 \mathrm{~nm}$ GNPs and LNP-GNPs in synchronized vs. asynchronous cell populations. Data are "mean \pm SD." for $n=3$. *Represents a statistically significant difference (unpaired $t$ test, $p<0.05$ )

\section{GNP-mediated radiation dose enhancement in synchronized vs. unsynchronized cell populations}

We noticed an improvement in radiation dose enhancement in synchronized cell populations treated with LNP-GNPs (see Fig. 6a). After a radiation dose of 2 Gy with $6 \mathrm{MeV}$ photons, the synchronized tumor cell population incorporated with LNP-GNPs produced a $27 \%$ enhancement in tumor cell death compared to the control (unsynchronized; no GNPs; 2 Gy). It is also evident that synchronization improved the radiation sensitization even in control cells with no NPs. This therefore clearly shows that a combination of LNP-GNPs and synchronization of the cell cycle could play a critical role in optimizing GNP-mediated radiation sensitization.

A small improvement in survival fraction after a single dose fraction could lead to a significant improvement in the overall treatment when many fractions are used over a period of time. Based on the experimental survival fractions for a single fraction of $2 \mathrm{~Gy}$ radiation dose (Fig. 6a), we estimated the improvement in GNP-mediated radiation dose enhancement for a typical treatment where 30 fractions were given over a period of time (see the table in Fig. 6b). For example, the addition of LNPs into synchronized tumor cells produced a 1000-fold enhancement in cell killing.

Monte Carlo (MC) simulations were performed to estimate the reduction in cell survival based on the experimental uptake data in Fig. 5b. As explained in the schematic diagram in Fig. 7a, the $5 \mathrm{~nm}$ GNPs were distributed uniformly in vesicles of diameter $50 \mathrm{~nm}$ (with an average of 8 GNPs per vesicle) within the cell cytoplasm. Our current GNP-LEM approach does not include changes in the cell shape and radiosensitivity for different cell phases. Instead, we consider the difference between synchronous and asynchronous cell populations by using the different number of GNPs in the cells. We accounted for both uncertainties of GNP uptake measurement and statistical uncertainty in the model. The results of the MC simulations (Fig. 7b) support the general trends of the experimental survival fraction data shown in Fig. 6a, but at a highly reduced rate. 


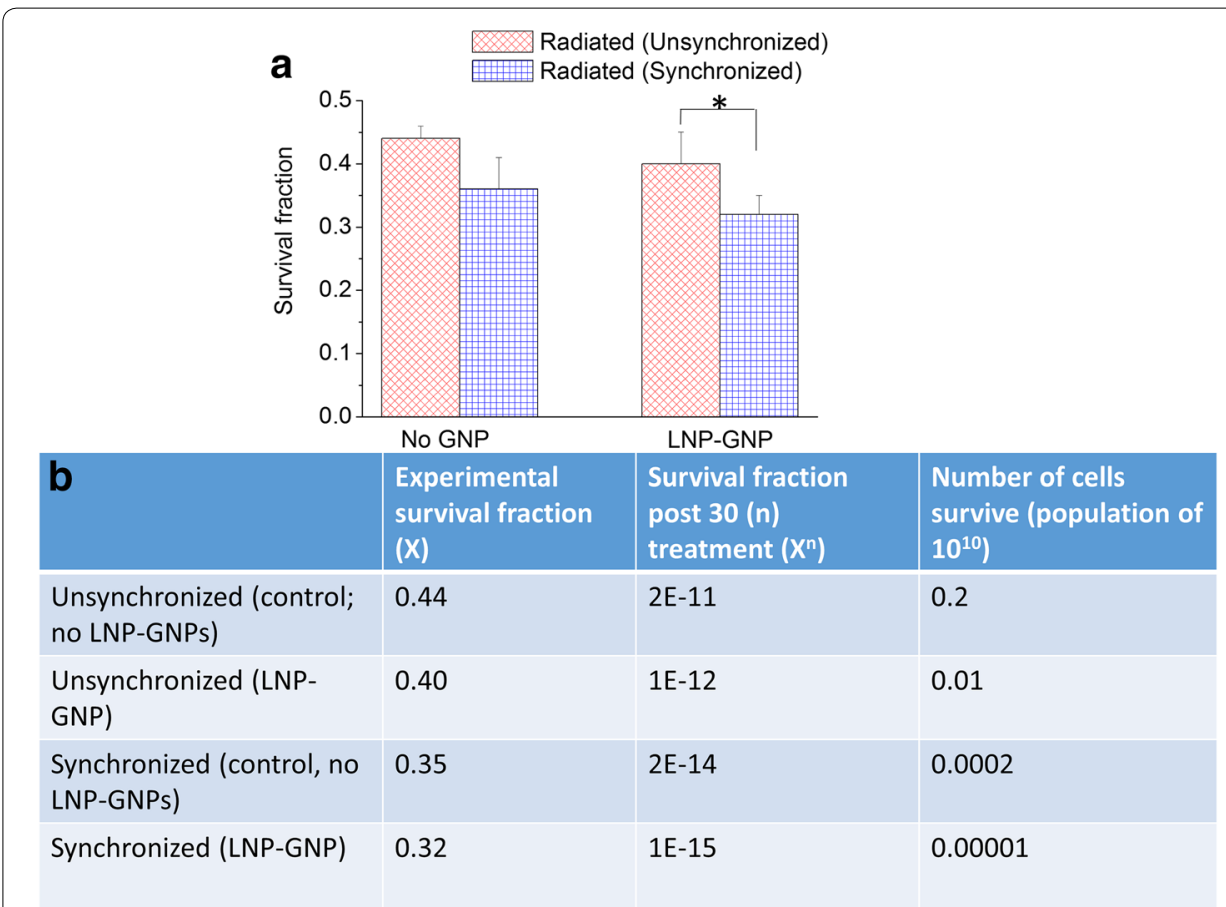

Fig. 6 GNP-mediated radiation therapy in a synchronized vs. unsynchronized (control) tumor cell population. a Experimental survival fractions of cells treated with $2 \mathrm{~Gy}$ radiation dose. Data are "mean $\pm S D^{\prime}$ for $n=3$. *Represents a statistically significant difference (unpaired $t$ test, $p<0.05$ ). b Estimation of the improvement in tumor cell killing for a hypothetical treatment plan based on the experimental survival fractions. A tumor population was given 30 fractions of $2 \mathrm{~Gy}$ dose over a period of time under different conditions: unsynchronized (no GNPs), unsynchronized (with LNP-GNPs), synchronized (no GNPs), and synchronized (with LNP-GNPS)

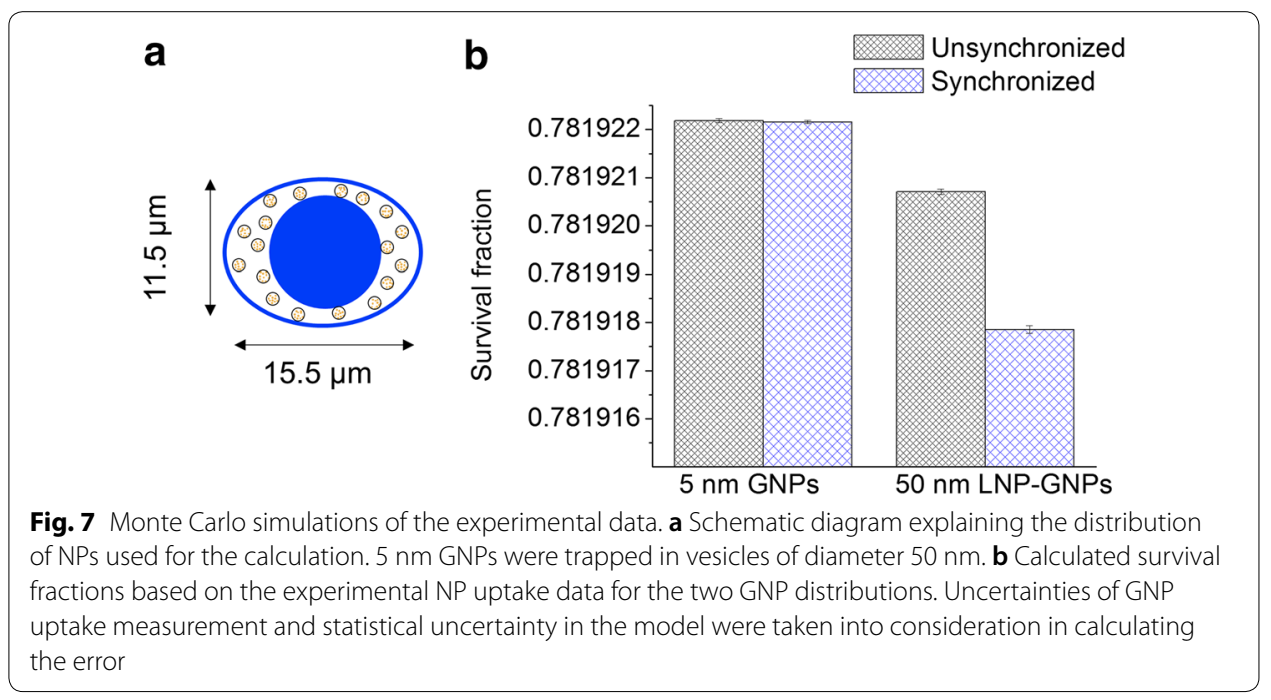

\section{Discussion}

The introduction of high-Z material systems such as GNPs into current radiation therapy protocols is being tested worldwide to enhance the local dose while reducing damage to nearby healthy tissue. This involves designing GNP complexes for optimized delivery in 
all three interfaces: monolayer, multilayer (tissue-like), and in vivo. Our LNP-GNP system has the following characteristics: (a) PEGylated for future in vivo studies, (b) can target low-density lipoprotein (LDL) receptors on tumor cells, (c) optimal size (50 nm) for uptake at the individual cell level, and d) improved residence time of smaller NPs for in vivo application. Using this LNP-GNP system, we could overcome some of the difficulties faced in the pioneering study by Hainfeld et al. where $1.9 \mathrm{~nm}$ GNPs were used to treat tumors in mice (Hainfeld et al. 2004). For example, the two major drawbacks of that study were as follows: (a) a very high concentration of NPs was necessary since very small NPs could not enter cells efficiently and (b) radiation treatment had to be carried out right after injection of NPs since the residence time of smaller NPs in the blood was limited by fast clearance through the renal system (Hainfeld et al. 2004).

It is essential for NPs to internalize within cells to reduce the concentration of GNPs necessary for radiosensitization. Studies have shown that the size of the NPs plays a critical role in their cellular internalization process (Chithrani 2010; Zhang et al. 2009; Akinc et al. 2010). Among the size range of 1-100 nm, the optimum size for uptake at the monolayer level is $50 \mathrm{~nm}$ (Chithrani 2010). Hence, in this study, we designed our LNP system to be $50 \mathrm{~nm}$ in diameter and used it as a Trojan horse to deliver small NPs of $5 \mathrm{~nm}$ diameter into tumor cells. We were able to improve the uptake of small $5 \mathrm{~nm}$ GNPs 39-fold through encapsulation in an LNP system (see Fig. 3b). In addition, the LNP system was functionalized to target LDL receptors. For example, PEG-lipids with short fatty acid chains (C14) were able to form a complex which could interact with the LDL receptor on the cell surface once ApoE in the tissue culture media adsorbs onto the LNP surface (Akinc et al. 2010; Kulkarni et al. 2017; Mui et al. 2013; Chen et al. 2016). It should be noted that the LDL receptor is almost ubiquitously expressed, albeit to different levels. For example, compared with another breast cancer cell line, MCF7 cells, LDL receptor is three- to fivefold higher in MDA-MB-231 cells (TNBC), and MDA-MB-231 cells exhibit high capacity and high affinity binding of LDL in comparison to MCF7 cells (Ye et al. 2016).

After the initial uptake studies, we used $6 \mathrm{MeV}$ photons for delivering the radiation treatment since they are the most commonly used radiation modality in cancer treatment. However, photons of energy in the $\mathrm{keV}$ range could produce higher sensitization in the presence of GNPs, mainly through the photoelectric effect. For example, MC simulation studies have shown that microscopic dose enhancement for $\mathrm{keV}$ photons can be increased by a factor of 3000 as compared to doses originating from a hypothetical water NP at distances of approximately $10 \mu \mathrm{m}$ (Lin et al. 2014; Jones et al. 2010). The dominant interaction with GNPs for MeV photon beams is through Compton scattering with a cross section three orders of magnitude lower than the photoelectric cross section for $\mathrm{keV}$ photons. However, $\mathrm{MeV}$ photons contain an increasing percent of $\mathrm{keV}$ photons and electrons with treatment depth which could lead to enhanced cell death in the presence of GNPs within cells, as illustrated in this study (Figs. 4, 6). In spite of the improvement in uptake of GNPs, it was not possible to generate a statistically significant radiation dose enhancement in tumor cells as illustrated in Fig. 4b. Therefore, we investigated whether the uptake and radiation dose enhancement due to GNPs can be further improved by synchronizing the tumor cell population. 
Synchronization of the tumor cell population resulted in a 73-fold enhancement in the uptake of $5 \mathrm{~nm}$ GNPs encapsulated within LNPs (Fig. 5). For example, $5 \mathrm{~nm}$ GNP uptake per cell increased from 1200 to 88,477 using the LNP-GNP system. We tested both the double thymidine block method and the serum starvation method as methods of cell synchronization. It was found that the double thymidine block method (DTBM) was more effective in cell cycle synchronization, as seen in Additional file 1: Fig. S5. After cell cycle synchronization, we introduced new media to cells for $3 \mathrm{~h}$ and then incubated them with NPs. Most of the cell population was in the G2/M phase at the time of NP incubation (see Fig. 5a). Previous research has shown that NP uptake can vary depending on the phase of the cell cycle chosen for synchronization and the NP uptake was ranked according to the different phases: G2/M > S > G0/G1 (Kim et al. 2011). Hence, the improved uptake of GNPs can be correlated with the previously published work related to silica NPs. This method is also clinically feasible since thymidine is an approved pharmacological agent which synchronizes cells in S phase (Heinemann et al. 2010). Based on our results, we pursued the GNP-mediated radiation therapy work with cells synchronized with DTBM (see Fig. 6).

The phase of the tumor cell population can play a big role in radiation therapy as well. A recent study has shown higher radiosensitivity during the $S / G 2 / M$ phase (Otani et al. 2016). Based on the data in Fig. 5a, a higher percent of the cell population was still in $\mathrm{S} /$ $\mathrm{G} 2 / \mathrm{M}$ at the time of radiation treatment as compared to the unsynchronized population. The incorporation of $5 \mathrm{~nm}$ GNPs into LNPs resulted in 27\% enhancement in tumor cell death in a synchronized tumor cell population after being irradiated with a single dose fraction of $2 \mathrm{~Gy}$ with $6 \mathrm{MeV}$ photons (see Fig. 6). The larger therapeutic effect due to LNP-GNPs is likely due to a combination of the cell cycle synchronization effect and the presence of GNPs during the radiation treatment. Experimental results were in agreement with the trends of MC-based modeling, but to a lesser extent (Fig. 7b). This could be due to the fact that some biological mechanisms were not yet incorporated into MC simulations. In addition, the simulations were purely based on localized physical dose enhancement, ignoring the potentially dominant effects GNPs have in the generation of reactive oxygen species and other cell stressors. In addition, we found no significant enhancement in DNA DSBs after administering a 2 Gy dose in the synchronized vs. unsynchronized population (see Additional file 1: Fig. S6). This indicates the potential importance of other mechanisms of cell damage including damage to other cellular organelles, such as mitochondria.

Furthermore, small differences in cell survival fractions after a single treatment can translate into significant differences in cell survival fractions after multiple treatments (Fig. 6b). This concept has been introduced by Hill and Bristow (Hill and Robert 2008). Using the experimental data shown in Fig. 6a, we estimated that the incorporation of LNP-GNPs into a synchronized tumor cell population would improve cell killing 1000fold for a clinical treatment plan where 30 fractions of 2 Gy radiation dose were given over a period of time. In addition, it is important to mention that our NP experiments were carried out using a clinically feasible $0.2 \mathrm{nM}$ concentration. 


\section{Conclusions}

A triple combination of the cell cycle synchronization, GNPs administration and radiation therapy has the potential to greatly improve outcomes in breast cancer therapy. Enhanced uptake and radiation sensitivity of a synchronized MDA-MB-231 triple-negative breast cell population produced a significant improvement in cell killing. For example, synchronizing cells and the addition of LNPs into tumor cells produced a 27\% enhancement in tumor cell death compared to the control (unsynchronized; no GNPs; 2 Gy). As estimated in this study, even a smaller improvement in cell death with a single dose fraction can lead to a significant improvement when multiple dosages are used over the course of treatment. Our ultimate goal is to incorporate anticancer drugs in addition to GNPs into the current LNP system for investigating GNP-mediated combined chemotherapy with radiation therapy (chemoradiation) (Yang et al. 2018). Hence, the design and development of lipid-NP-based GNP delivery systems was motivated by the goal to create new materials and devices with superior properties, functions, efficiencies and safety in future cancer nanomedicine. We believe that a combination of cell cycle synchronization, GNPs, radiation therapy and anticancer drugs would provide further synergistic effects in optimizing GNP-mediated cancer treatment in the near future.

\section{Additional file}

Additional file 1. Additional figures.

\section{Abbreviations}

BC: breast cancer; DMEM: Dulbecco's modified Eagle's medium; DNA: deoxyribonucleic acid; DSBs: double-strand breaks; DTBM: double thymidine block method; GNPs: gold nanoparticles; HSI: hyperspectral imaging; ICP-MS: inductively coupled plasma mass spectroscopy; keV: kiloelectron voltage; LDL: low-density lipoprotein; LNP: lipid nanoparticles; MeV: megaelectron voltage; MC: Monte Carlo; PEG: polyethylene glycol; PBS: phosphate-buffered saline; TEM: transmission electron microscopy; TNBC: triple-negative breast cancer; UV: ultraviolet.

\section{Authors' contributions}

All authors listed have made substantial, direct and intellectual contributions to the work discussed in this publication. KB, KR, DC, JS and PC designed the article. KB, KR, CO, JK and WS performed the experiments analyzed the data, and drafted the paper. All authors discussed the data and revised the manuscript. All authors read and approved the final manuscript.

\section{Author details}

${ }^{1}$ Department of Physics and Astronomy, University of Victoria, Victoria, BC, Canada. ${ }^{2}$ Department of Biochemistry and Molecular Biology, University of British Columbia, Vancouver, BC, Canada. ${ }^{3}$ Department of Medical Genetics, BC Children's Hospital Research Institute, University of British Columbia, Vancouver, BC, Canada. ${ }^{4}$ Department of Biochemistry and Microbiology, University of Victoria, Victoria, Canada. ${ }^{5}$ Massachusetts General Hospital \& Harvard Medical School, Boston, MA, USA. ${ }^{6}$ British Columbia Cancer Agency, Victoria, BC, Canada.

\section{Acknowledgements}

We would like to acknowledge the research support from Dr. Wayne Beckham and Dr. Perry Howard at British Columbia Cancer Agency (BCCA) and University of Victoria.

\section{Competing interests}

All authors declare that they have had no financial relationships with any organizations that might have an interest in the submitted work and the authors have no other relationships or activities that could influence the submitted work.

\section{Availability of data and materials}

All data generated or analyzed during this study are included in this published article (and its additional information on file).

\section{Funding}

This work was supported by the Natural Sciences and Engineering Research Council of Canada (NSERC), British Columbia Cancer Agency (BCCA), University of Victoria, Canada Foundation for Innovation (CFI), and in part by the National Institutes of Health (NIH)/National Cancer Institute (NCl) Grant R01 CA187003. 


\section{Publisher's Note}

Springer Nature remains neutral with regard to jurisdictional claims in published maps and institutional affiliations.

Received: 30 December 2018 Accepted: 6 April 2019

Published online: 25 April 2019

\section{References}

Abolfazli MK, Mahdavi SR, Ataei G. Studying effects of gold nanoparticle on dose enhancement in megavoltage radiation. J Biomed Phys Eng. 2015;5:185-90.

Akinc A, Querbes W, De S, Qin J, Frank-Kamenetsky M, Jayaprakash KN, Jayaraman M, Rajeev KG, Cantley WL, Dorkin JR, Butler JS. Targeted delivery of RNAi therapeutics with endogenous and exogenous ligand-based mechanisms. Mol Ther. 2010;18(7):1357-64.

Arthur DW, Vicini FA, Wazer DE, Khan AJ. SpringerLink, short course breast radiotherapy: a comprehensive review of hypofractionation, partial breast, and intra-operative irradiation. 1st ed. Springer: International Publishing; 2016.

Baowan D, Thamwattana N. Modelling encapsulation of gold and silver nanoparticles inside lipid nanotubes. Physica A. 2014:396:149-54

Boudailffa B, Cloutier P, Hunting D, Huels MA, Sanche L. Resonant formation of DNA strand breaks by low-energy (3 to $20 \mathrm{eV}$ ) electrons. Science. 2000;287:1658-60.

Butterworth KT, McMahon SJ, Taggart LE, Prise KM. Radiosensitization by gold nanoparticles: effective at megavoltage energies and potential role of oxidative stress. Transl Cancer Res. 2013;2:269-79.

Carter JD, Cheng NN, Qu Y, Suarez GD, Guo T. Nanoscale energy deposition by X-ray absorbing nanostructures. J Phys Chem B. 2007;111:11622-5.

Chang DS, Lasley FD, Das IJ, Mendonca MS, Dynlacht JR. SpringerLink, basic radiotherapy physics and biology. Springer: International Publishing; 2014.

Chen S, Tam YYC, Lin PJC, Sung MMH, Tam YK, Cullis PR. Influence of particle size on the in vivo potency of lipid nanoparticle formulations of siRNA. J Control Release. 2016;235:236-44.

Chithrani DB. Intracellular uptake, transport, and processing of gold nanostructures. Mol Membr Biol. 2010;27:299-311.

Chithrani BD, Ghazani AA, Chan WCW. Determining the size and shape dependence of gold nanoparticle uptake into mammalian cells. Nano Lett. 2006;6:662-8.

Chithrani DB, Jelveh S, Jalali F, Prooijen MV, Allen C, Bristow RG, Hill RP, Jaffray DA. Gold nanoparticles as radiation sensitizers in cancer therapy. Radiat Res. 2010;173:719-28.

Cho SH, Krishnan S. Cancer nanotechnology: principles and applications in radiation oncology. Boca Raton: CRC Press; 2013.

Cruje C, Yang C, Uertz J, van Prooijen M, Chithrani BD. Optimization of PEG coated nanoscale gold particles for enhanced radiation therapy. RSC Adv. 2015;5(123):101525-32.

DeSantis CE, Ma J, Goding Sauer A, Newman LA, Jemal A. Breast cancer statistics, 2017, racial disparity in mortality by state. CA Cancer J Clin. 2017;67(6):439-48.

Hainfeld JF, Slatkin DN, Smilowitz HM. The use of gold nanoparticles to enhance radiotherapy in mice. Phys Med Biol. 2004;49:N309.

Heinemann L, Simpson GR, Annels NE, Vile R, Melcher A, Prestwich R, Harrington KJ, Pandha HS. The effect of cell cycle synchronization on tumor sensitivity to reovirus oncolysis. Mol Ther. 2010;18:2085-93.

Hill RP, Robert B. The scientific basis of radiotherapy. In: Tannock IF, Richard P, Robert G, Harrington L, editors. The basic science of oncology. Toronto: McGraw-Hill; 2008. p. 289-321.

Jain S, Coulter JA, Hounsell AR, Butterworth KT, MCMahon SJ, Hyland WB, Muir MF, Dickson GR, Prise KM, Currell FJ. Cellspecific radiosensitization by gold nanoparticles at megavoltage radiation energies. Int J Radiat Oncol Biol Phys. 2011;79:531-9.

Jones BL, Krishnan S, Cho SH. Estimation of microscopic dose enhancement factor around gold nanoparticles by Monte Carlo calculations. Med Phys. 2010;37:3809-16.

Kang JH, Ko YT. Lipid-coated gold nanocomposites for enhanced cancer therapy. Int J Nanomed. 2015;10:33-45.

Kim JA, Åberg C, Salvati A, Dawson KA. Role of cell cycle on the cellular uptake and dilution of nanoparticles in a cell population. Nat Nanotechnol. 2011:7:62

Kulkarni JA, Myhre JL, Chen S, Tam YYC, Danescu A, Richman JM, Cullis PR. Design of lipid nanoparticles for in vitro and in vivo delivery of plasmid DNA. Nanomedicine. 2017;13:1377-87.

Kulkarni JA, Darjuan MM, Mercer JE, Chen S, van der Meel R, Thewalt JL, Tam YYC, Cullis PR. On the formation and morphology of lipid nanoparticles containing ionizable cationic lipids and siRNA. ACS Nano. 2018;12:4787-95.

Lee P, Zhang RZ, Li V, Liu XL, Sun RWY, Che CM, Wong KKY. Enhancement of anticancer efficacy using modified lipophilic nanoparticle drug encapsulation. Int J Nanomed. 2012;7:731-7.

Leung AKK, Tam YYC, Chen S, Hafez IM, Cullis PR. Microfluidic mixing: a general method for encapsulating macromolecules in lipid nanoparticle systems. J Phys Chem B. 2015;119:8698-706.

Lin Y, McMahon SJ, Scarpelli M, Paganetti H, Schuemann J. Comparing gold nano-particle enhanced radiotherapy with protons, megavoltage photons and kilovoltage photons: a Monte Carlo simulation. Phys Med Biol. 2014;59:7675-89.

Lin Y, McMahon SJ, Paganetti H, Schuemann J. Biological modeling of gold nanoparticle enhanced radiotherapy for proton therapy. Phys Med Biol. 2015;60:4149-68.

Liu Z, Lee Y, Jang JH, Li Y, Han X, Yokoi K, Ferrari M, Zhou L, Qin L. Microfluidic cytometric analysis of cancer cell transportability and invasiveness. Sci Rep. 2015;5:14272.

Mui BL, Tam YK, Jayaraman M, Ansell SM, Du X, Tam YYC, Lin PJ, Chen S, Narayanannair JK, Rajeev KG, Manoharan M, Akinc A, Maier MA, Cullis P, Madden TD, Hope MJ. Influence of polyethylene glycol lipid desorption rates on pharmacokinetics and pharmacodynamics of siRNA lipid nanoparticles. Mol Ther. 2013;2:e139. 
O'Neill P. The chemical basis of radiation biology. Int J Radiat Biol Relat Stud Phys Chem Med. 1987;52:976.

Otani K, Naito Y, Sakaguchi Y, Seo Y, Takahashi Y, Kikuta J, Ogawa K, Ishii M. Cell-cycle-controlled radiation therapy was effective for treating a murine malignant melanoma cell line in vitro and in vivo. Sci Rep. 2016;6:30689.

Schuemann J, McNamara AL, Ramos-Méndez J, Perl J, Held KD, Paganetti H et al(2019) TOPAS-nBio: an extension to the TOPAS simulation toolkit for cellular and subcellularradiobiology. Radiat Res. 191(2):125-138. https://doi. org/10.1667/RR15226.1

Sung W, Ye S-J, McNamara AL, McMahon SJ, Hainfeld J, Shin J, Smilowitz HM, Paganetti H, Schuemann J. Dependence of gold nanoparticle radiosensitization on cell geometry. Nanoscale. 2017;9:5843-53.

Von White G, Chen Y, Roder-Hanna J, Bothun GD, Kitchens CL. Structural and thermal analysis of lipid vesicles encapsulating hydrophobic gold nanoparticles. ACS Nano. 2012;6:4678-85.

Wolfe T, Chatterjee D, Lee J, Grant JD, Bhattarai S, Tailor R, Goodrich G, Nicolucci P, Krishnan S. Targeted gold nanoparticles enhance sensitization of prostate tumors to megavoltage radiation therapy in vivo. Nanomedicine. 2015;11:1277-83.

Yang C, Bromma K, Chithrani BD. Peptide mediated in vivo tumor targeting of nanoparticles through optimization in single and multilayer in vitro cell models. Cancers. 2018a;10:84.

Yang C, Bromma K, Sung W, Schuemann J, Chithrani D. Determining the radiation enhancement effects of gold nanoparticles in cells in a combined treatment with cisplatin and radiation at therapeutic megavoltage energies. Cancers. 2018b;10:150

Ye J, Xia X, Dong W, Hao H, Meng L, Yang Y, Wang R, Lyu Y, Liu Y. Cellular uptake mechanism and comparative evaluation of antineoplastic effects of paclitaxel-cholesterol lipid emulsion on triple-negative and non-triple-negative breast cancer cell lines. Int J Nanomed. 2016;11:4125-40.

Zhang S, Li J, Lykotrafitis G, Bao G, Suresh S. Size-dependent endocytosis of nanoparticles. Adv Mater. 2009;21:419-24.

Ready to submit your research? Choose BMC and benefit from:

- fast, convenient online submission

- thorough peer review by experienced researchers in your field

- rapid publication on acceptance

- support for research data, including large and complex data types

- gold Open Access which fosters wider collaboration and increased citations

- maximum visibility for your research: over $100 \mathrm{M}$ website views per year

At BMC, research is always in progress.

Learn more biomedcentral.com/submissions 